\title{
Efecto del consumo de polimnia sonchifolia (yacón) sobre los niveles de lípidos en estu- diantes con sobrepeso y obesidad de una universidad privada, Lima, 2012
}

\author{
Pedro PÉREZ FERNÁNDEZ*, Marleni BARAHONA GRANDES, Lizbeth ORIHUELA MEZA, \\ y Dennise VENEGAS CÁRDENAS \\ EAP Nutrición, Facultad de Ciencias de la Salud, Universidad Peruana Unión, Alt. Km 19; Carretera Central, Lurigancho, Lima
}

\begin{abstract}
RESUMEN
El objetivo del estudio fue determinar los efectos del consumo de polimnia sonchifolia (Yacón) sobre los niveles de lípidos en estudiantes con sobrepeso y obesidad. Estudio de tipo pre experimental, diseño pre prueba y post prueba con un solo grupo, de corte longitudinal. La muestra fueron jóvenes de edades entre 18-30 años que residen en la universidad privada, siendo una total de 41 estudiantes, de los cuales 27 fueron damas y 14 varones. El tipo de muestreo fue no probabilístico por conveniencia, a quienes durante 30 días se les entregó $150 \mathrm{~g}$. de yacón para el consumo en ayunas. Luego se realizó el perfil lipídico en sangre por laboratorios DEJER, para los niveles de colesterol total, colesterol LDL, HDL, y triglicéridos. Asimismo, se realizó la evaluación antropométrica: peso y talla. El análisis de datos utilizado fue la prueba " $\mathrm{t}$ " de Student con un nivel de significancia de $\alpha=0.05 \%$. La media del colesterol total plasmático en las personas antes de consumir el yacón fue de $199.5122 \mathrm{mg} / \mathrm{dl}$. Y al término de la investigación la media del colesterol total plasmático de las personas fue $186.1220 \mathrm{mg} / \mathrm{dl}$. Llegando a la conclusión que el consumo de Yacón, reduce el colesterol significativamente.
\end{abstract}

Palabras clave: Yacón, triglicéridos, colesterol, HDL, LDL, obesidad, sobrepeso.

\begin{abstract}
The aim of the study was to determine the effects of consumption of Polimnia sonchifolia (Yacon) on lipid levels in overweight and obese students. Study pre-type experimental design pre test and post test with a single group, slitting. The sample were young people aged between 18-30 years living in private universities. be a total of 41 students, of whom 27 were women and 14 men. The sampling was not probabilistic convenience, who for 30 days were given $150 \mathrm{~g}$. consumption of yacon for fasting. Then there was the blood lipid profile DEJER laboratories, for levels of total cholesterol, LDL, HDL, and triglycerides. Likewise Anthropometric assessment: weight and height. The data analysis used was the " $\mathrm{t}$ " Student with a significance level of $\alpha=0.05 \%$. The mean plasma total cholesterol in people before consuming the yacon was $199.5122 \mathrm{mg} / \mathrm{dl}$. And at the end of the investigation the average plasma cholesterol of people was $186.1220 \mathrm{mg} / \mathrm{dl}$. Concluding that the consumption of yacon, reduces cholesterol significantly
\end{abstract}

Keywords: Yacon, triglycerides, cholesterol, HL, LDL, obesity, overweight.

*Correspondencia: peter 1788@hotmail.com 


\section{INTRODUCCIÓN}

Las enfermedades cardiovasculares (ECVs) representa la primera causa de muerte, pues provoca una muerte por cada tres fallecidos y lamentablemente su incidencia aumenta en países en desarrollo a medida que mejora la situación económica y el nivel de bienestar. En los países en vías de desarrollo, comienzan a aumentar la Diabetes, la Obesidad, la Hipertensión y las Dislipidemias, entre otros factores que contribuyen a las enfermedades cardiovasculares. Para el año 2020 se calcula que las muertes por ECVs se incrementarán en un $29 \%$ en mujeres y un $48 \%$ en varones en los países ricos, mientras que en los países en desarrollo, en un lapso de 30 años, las muertes por cardiopatías habrán aumentado un $20 \%$ en mujeres y un $13 \%$ en varones (OMS, 2009).

Existe una estrecha correlación entre ECVs y altos niveles de lípidos sanguíneos. Los trastornos lipídicos pueden incrementar el riesgo de cardiopatía isquémica en hombres y en mujeres, probablemente por su asociación con otros factores de riesgo como la diabetes mellitus tipo 2, obesidad y la hipertensión arterial (Gotto, 2001).

En el Perú, la Encuesta Nacional de Indicadores Nutricionales, Bioquímicos, Socioeconómicos y Culturales relacionados con las Enfermedades Crónico Degenerativas (2006), mostró que el sobrepeso afectaba al $35.3 \%$ de la población peruana $(39.1 \%$ en los mayores de 60 años); mientras que la obesidad, al 16,5\% de la población (16,8\% en los mayores de 60 años). Con respecto a la prevalencia de hipertrigliceridemia y contenido elevado de LDL, mostró que afectaba aproximadamente al 15\% de la población. La prevalencia de hipertrigliceridemia se incrementaba con la edad, siendo los más afectados los pobladores de 50 años. La prevalencia de hipercolesterolemia y prevalencia de LDL era mayor en mujeres (22\%). Mostró además una baja prevalencia de niveles anormales de colesterol HDL $(<35 \mathrm{mg} / \mathrm{dl})$ en la población evaluada.

El Yacón (polymnia sonchifolia) raíz andina se consume fresco, es dulce y es cortado para poder consumir en ensaladas dando sabor y textura. Pertenece al grupo de raíces y tubérculos andinos (RTA), tiene un alto contenido de inulina y fructoologosacaridos (FOS). Nutricionalmente el yacón posee un valor calórico bajo por su alto contenido de agua y carbohidratos (19,9\%). Presenta un alto contenido de fructoologosacáridos entre 60 a $70 \%$ en base seca y una baja proporción de azúcares reductores como glucosa, fructosa y sucrosa (Araya, 1995). Así como lo muestra el estudio realizado por Collantes (2009), que tuvo como objetivo determinar el efecto del consumo de polimnia sonchifolia (yacón) en los niveles de lípidos en pacientes dislipidémicos, obteniendo resultados en el grupo experimental donde al inicio del estudio tuvieron un promedio 276.7 $\mathrm{mg} / \mathrm{dl}$ de colesterol y al finalizar el estudio disminuyeron a $230.1 \mathrm{mg} / \mathrm{dl}$.

El estudio tuvo como objetivo determinar los efectos del consumo de polymnia sonchifolia (yacón) sobre los niveles de dislipidemia en estudiantes de una universidad privada.

\section{MATERIAL Y MÉTODOS}

Tipo de estudio pre-experimental porque existió manipulación de las variables y luego se observó la determinación del efecto. Longitudinal: porque tuvo una base de datos antes y después a fin de analizar el efecto del consumo de yacón en los niveles de lípidos de estudiantes en sobrepeso y obesidad realizado en residencias de varones y señoritas de la universidad privada. Se manejó algunos criterios de inclusión y exclusión como residentes internos basados en consumo de dietas completas, estabilidad fisiológica y edades entre 18 y 30 años mientras no se incluían aquellos mayores de 30 años y aquellos que tengan dietas rigurosas: hipograsa, hipercalórico, hipocalórica, hiperproteica, hipo proteica y con disfunciones fisiológicas, la comprendida por 41 estudiantes 27 varones y 14 mujeres en estado de sobrepeso y obesidad. La ración de yacón fue de $150 \mathrm{gr}$, pelado cortado en trozos, envasado en taperes y embolsado para ser distribuido a los participantes. Las raíces utilizadas fueron aquéllas con buena turgencia y aparentes descartándose aquellas sin esta condición. Se consideró los criterios inclusión-exclusión para las condiciones de muestreo en los cuales se realizó el análisis del perfil lipídico mediante muestra de sangre por medio del laboratorio DEJER, para determinar los niveles de colesterol total, colesterol LDL, HDL, y triglicéridos. La toma de la primera muestra de sangre fue el 7 de mayo y la segunda toma de muestra de sangre fue el 8 de junio del 2012. Asimismo se realizó la evaluación antropométrica como peso con una balanza de pie marca "Detecto" con precisión aproximada a 50 gramos, donde el participante se ubica en la balanza sobre el centro de la plataforma con un mínimo de ropa. Con respecto a la talla se utilizó el tallímetro con escala de pared y plano de broca de modo que la cabeza debe estar orientada en el plano de Frankfurt (una línea paralela al piso une el reborde óseo inferior de la órbita con el conducto auditivo externo). Con una aproximación a $5 \mathrm{~mm}$. Cabe mencionar que durante el transcurso de la investigación, ninguno de los participantes recibieron recomendación alguna para reducir el colesterol, como: orientación dietética (aumento de ciertos alimentos y reducción de alimentos ricos en grasas o carbohidratos refinados), práctica de actividad 
física, no comer de noche, etc. La única diferencia fue el consumo de $150 \mathrm{~g}$ de yacón en ayunas, por lo demás no se quiso interferir con el estilo de vida propio de cada participante.

Una vez recolectada la información se transfirió a la matriz de datos del software para su respectivo análisis utilizando el software estadístico SPSS 11.0 y con estos análisis se realizó la comparación de datos mediante la prueba "t" de Student de diferencia de medias en grupos independientes para probar si existe diferencia significativa en los tipos de colesterol, el nivel de significancia fue de $\alpha=0.05 \%$.

\section{RESULTADOS}

\section{Tabla 1}

Medias del colesterol total plasmático en el grupo experimental al inicio y al final del efecto del consumo de Polimnia Sonchifolia (yacón) sobre los niveles de lípidos en estudiantes con sobrepeso y obesidad

\begin{tabular}{lcccc}
\hline & Media & N & Desviación típ. & $\begin{array}{c}\text { Error típ. de la } \\
\text { media }\end{array}$ \\
\hline Colesterol Total (INICIO) & 199,5122 & 41 & 25,38220 & 3,96403 \\
Colesterol Total (FINAL) & 186,1220 & 41 & 22,65413 & 3,53798 \\
\hline
\end{tabular}

Se observa en la tabla 1, la media del colesterol total plasmático en las personas antes de consumir el yacón fue de $199.5122 \mathrm{mg} / \mathrm{dl}$. Y al término de la investigación la media del colesterol total plasmático de las personas fue $186.1220 \mathrm{mg} / \mathrm{dl}$.

Tabla 2

Prueba $T$ para colesterol HDL plasmático en el grupo experimental al inicio y al final del efecto del consumo de Polimnia Sonchifolia (yacón) sobre los niveles de lípidos en estudiantes con sobrepeso y obesidad

\begin{tabular}{|c|c|c|c|c|c|c|c|c|}
\hline & \multicolumn{5}{|c|}{ Diferencias relacionadas } & \multirow{2}{*}{$\frac{\mathrm{t}}{\text { Media }}$} & \multirow{2}{*}{$\begin{array}{c}\text { gl } \\
\begin{array}{l}\text { Desvia- } \\
\text { ción típ. }\end{array}\end{array}$} & \multirow{2}{*}{$\begin{array}{c}\begin{array}{c}\text { Sig. } \\
\text { (bilateral) }\end{array} \\
\text { Error } \\
\text { típ. de la } \\
\text { media }\end{array}$} \\
\hline & Media & $\begin{array}{l}\text { Desvia- } \\
\text { ción típ. }\end{array}$ & $\begin{array}{l}\text { Error } \\
\text { típ. de la } \\
\text { media }\end{array}$ & $\begin{array}{r}95 \% \text { In } \\
\text { confianza } \\
\text { re }\end{array}$ & $\begin{array}{l}\text { valo de } \\
\text { ra la dife- } \\
\text { a }\end{array}$ & & & \\
\hline & Inferior & Superior & Inferior & Superior & Inferior & Superior & Inferior & Superior \\
\hline $\begin{array}{c}\text { HDL } \\
\text { (Inicio) } \\
\text { HDL } \\
\text { (Final) }\end{array}$ & 3,19512 & 4,77608 & ,74590 & 1,68760 & 4,70264 & 4,284 & 40 &, 000 \\
\hline
\end{tabular}

La tabla 2 muestra si existe diferencia significativa del colesterol HDL plasmático inicial y final $($ Sig. $=0,00)$, por lo que se puede afirmar que el consumo de yacón logró disminuir significativamente los niveles de colesterol HDL plasmático.

Tabla 3

Medias del colesterol LDL plasmático en el grupo experimental al inicio y al final del efecto efecto del consumo de Polimnia Sonchifolia (yacón) sobre los niveles de lípidos en estudiantes con sobrepeso y obesidad de la universidad peruana unión, 2012

\begin{tabular}{ccccc}
\hline & Media & $\mathrm{N}$ & Desviación típ. & Error típ. de la media \\
\hline LDL (Inicio) & 113,9854 & 41 & 17,70776 & 2,76549 \\
& 106,8537 & 41 & 15,90057 & 2,48325 \\
\hline
\end{tabular}


La tabla 3 muestra la media del colesterol LDL plasmático en los participantes antes de consumir el yacón fue de $113,98 \mathrm{mg} / \mathrm{dl}$, y al final, la media del colesterol LDL plasmático de los participantes fue 106,85 mg/dl. Observándose una disminución del promedio.

\section{Tabla 4}

Medias del triglicéridos en el grupo experimental al inicio y al final del efecto del consumo de polimnia sonchifolia (yacón) sobre los niveles de lípidos en estudiantes con sobrepeso y obesidad de la Universidad Peruana Unión, 2012

\begin{tabular}{ccccc}
\hline & Media & N & Desviación típ. & \multicolumn{2}{c}{ Error típ. de la } \\
media
\end{tabular}

En la tabla 4, se observa que la media de triglicéridos en los participantes antes de consumir el yacón fue de 141,46 $\mathrm{mg} / \mathrm{dl}$, y al final la media del colesterol LDL plasmático de los participantes fue 129,48 mg/dl. Lo que se confirma estadísticamente con la prueba $t$ de student, donde existe diferencia significativa en triglicéridos inicial y final $($ Sig. $=0,00)$, por lo que se puede afirmar que el consumo de yacón logró disminuir significativamente los niveles de triglicéridos.

\section{CONCLUSIONES}

- $\quad$ El consumo de yacón, redujo el colesterol significativamente de 199,51 mg/dl a $186.12 \mathrm{mg} / \mathrm{dl}$.

- Con respecto al colesterol HDL el consumo de yacón también disminuyó significativamente de (colesterol HDL de inicio $=56.68 \mathrm{mg} /$ dl. y colesterol HDL final $=53,48 \mathrm{mg} / \mathrm{dl}$.).
- El colesterol LDL con el consumo de Yacón se logró recudir significativamente (colesterol LDL de inicio $=113.98 \mathrm{mg} / \mathrm{dl}$. y colesterol LDL final $=$ $106.85 \mathrm{mg} / \mathrm{dl}$.$) .$

- Con respecto a los triglicéridos se puede decir que el consumo de Yacón logro recudir significativamente de (triglicéridos de inicio = 141,46 mg/dl. y triglicéridos final $=129,48 \mathrm{mg} / \mathrm{dl}$.).

\section{REFERENCIAS BIBLIOGRÁFICAS}

1. Araya C. (1995). La dieta primera línea de defensa para reducir el colesterol. Revista Chilena de Nutrición. N- 1 pp. 7.

2. Collantes; M. (2005). Efecto del consumo de polymina sonchifolia (yacón) en los niveles de lípidos en pacientes dislipidémico del Centro Geriátrico del Hospital Naval, Revista Científica de Ciencias de la Salud, vol 3 N 3. Universidad Peruana Unión. pp.10-18.
3. Gotto Antonio, M. Conceptos actuales en el diagnóstico y en el tratamiento de las dislipidemias. 2da edición 2001, pp. 99 - 102.

4. Organización Mundial de la Salud. (2009). World Health Report: Reducingrisks, promoting healthy life. Recuperado de Geneva: WHO: http://www. paho.org 